\title{
Sudden death in epilepsy: of mice and men
}

\author{
Daniel Friedman, MD,1 Janice Chyou, MD,2 and Orrin Devinsky, MD1 \\ ${ }^{1}$ Department of Neurology and 2Department of Cardiology, New York University School of Medicine, New York, New York, USA.
}

\begin{abstract}
A 20-year-old man with intellectual disability and intractable multifocal epilepsy presented to a neurologist for further evaluation and management. His seizures began at 4 months, the night after his first DPT vaccine, and he continued to have frequent tonic-clonic seizures throughout his life. Several weeks after his visit, he was found facedown on the floor, dead, by his family. His autopsy was unremarkable, but genetic testing revealed a frame shift mutation in $S C N 1 A$, consistent with severe myoclonic epilepsy of infancy (Dravet syndrome).
\end{abstract}

Sudden unexpected death in epilepsy (SUDEP) remains a silent killer - often occurring in sleep when patients are alone and frequently eluding recognition by families, clinicians, coroners, and medical examiners. SUDEP is the death of a person with epilepsy that is not due to trauma, drowning, status epilepticus, suicide, or other known causes of epilepsy-related mortality. There is often evidence of an associated seizure, but the patient is usually found dead in the morning (1). In one 40-year cohort study of childhood-onset epilepsy, SUDEP caused $38 \%$ of all deaths (2). It is estimated that SUDEP kills more people ( $3300 /$ year $)$ in the United States than fires $(-3100)$ or sudden infant death syndrome (SIDS) ( 2100) (3).

A variety of mechanisms likely cause SUDEP. Cases observed in the hospital and community have identified respiratory dysfunction, failure of protective reflexes, and cardiac arrhythmias (Figure 1 and ref. 1). Nearly all witnessed SUDEPs follow a terminal seizure, typically a generalized tonic-clonic seizure (GTCS). In case-control studies, the greatest risk factor for SUDEP was frequent GTCS (4). Aside from seizure control (5), little is known about how to prevent SUDEP.

Dravet syndrome (DS), or severe myoclonic epilepsy of infancy, is a rare genetic disorder usually due to mutations in the gene encoding a voltage-gated sodium channel, SCN1A. DS patients present in the first year of life with severe treatmentresistant epilepsy, intellectual disability, and gait disorder, and have a greater risk of premature death. Among DS children, the

Conflict of interest: The authors have declared that no conflict of interest exists.

Citation for this article: J Clin Invest. 2013; 123(4):1415-1416. doi:10.1172/JCI67759. estimated risk of SUDEP is $6 \%$ per decade (6). In contrast, in community cohorts of children with epilepsy, SUDEP occurs in approximately $0.2 \%$ per decade (1). This 30 -fold increase is a call to action to find the cause(s) of SUDEP in DS, identify highrisk patients, and translate mechanism into preventive strategies.

Kalume and colleagues' characterization of SUDEP in a mouse model is an enormous step forward, uncovering mechanisms and suggesting preventive therapy (7). Similar to SUDEP in patients without DS, these mice died after GTCS, especially when there were multiple seizures. However, unlike human SUDEPs captured on video-EEG, where postictal respiratory dysfunction and electrocerebral suppression are the most common apparent culprits (1), the DS mouse SUDEPs appeared to result primarily from asystole due to seizure-related parasympathetic hyperactivity. Treatment with a muscarinic receptor competitive antagonist reduced the incidence of SUDEP in DS mice. These investigators further refined the mechanism with selective knockouts, demonstrating that brain, rather than cardiac SCN1A, mediated SUDEP. These results point to defective neurocardiac coupling. It is still unclear whether the ictal parasympathetic hyperactivity in the Scn1a-mutant mice was a direct result of GABAergic neuronal $\mathrm{Na}^{+}$ current loss, the consequence of adaptive responses, or caused by changes in autonomic centers resulting from frequent, recurrent seizures.

Basic science rarely provides clinicians with such a clear target, yet the gap between mice and humans and between benches and bedsides remains great. Why do these mice suffer SUDEP in a relatively restricted period of youth, unlike humans with DS, for whom the risk of SUDEP persists? Is ictal parasympathetic hyperactivity and bradycardia a major cause of SUDEP in human DS? Ictal asystole and significant bradycardia occur in approximately $1 \%$ of epilepsy patients, but in a small series of patients who were implanted with pacemakers for ictal asystole, no devices were activated over a mean 5-year follow-up period (8). Thus, ictal asystole appears to be benign in adults with epilepsy. Furthermore, refractory epilepsy patients show excessive sympathetic activation between, during, and after seizures (9). If the DS mouse findings are confirmed in human DS, the generalizability to all SUDEP is unclear.

Sadly, the mechanism of death in human SUDEP - DS or otherwise remains poorly understood. Do nonfatal seizures in DS provide phenocopies of physiological changes in fatal seizures? We don't know. Without biomarkers for SUDEP in DS patients, it would be premature to extend these murine findings to humans. In the meantime, we should focus resources on education and therapies to reduce GTCS frequency and other SUDEP risk factors.

The limitation of this study is what was not studied. Despite elegant experiments suggesting that cardiac parasympathetic hyperactivity is part of the terminal cascade, doubt remains whether it is sufficient to cause SUDEP in DS. Could a sympathetic rebound cause the terminal changes as suggested by some of the authors' data (Figure 4A; ref. 7)? What about the brain and the lungs? Could seizure-induced brain changes outside of the medullary cardioregulatory nuclei contribute to SUDEP in these mice? Kalume and colleagues' mice had postictal EEG suppression, but they do not discuss its potential role. What roles do central and peripheral respiratory function play? And what of serotonergic, adrenergic, and purinergic neurotransmitters that have been implicated in other models of SUDEP (10)? Future animal studies should record multiple physiological parameters, including serum $\mathrm{O}_{2}$ and $\mathrm{CO}_{2}$ levels, respiratory rate, and cardiac and pulmonary histology. Perhaps the most important question raised by this study for clinicians caring for patients 


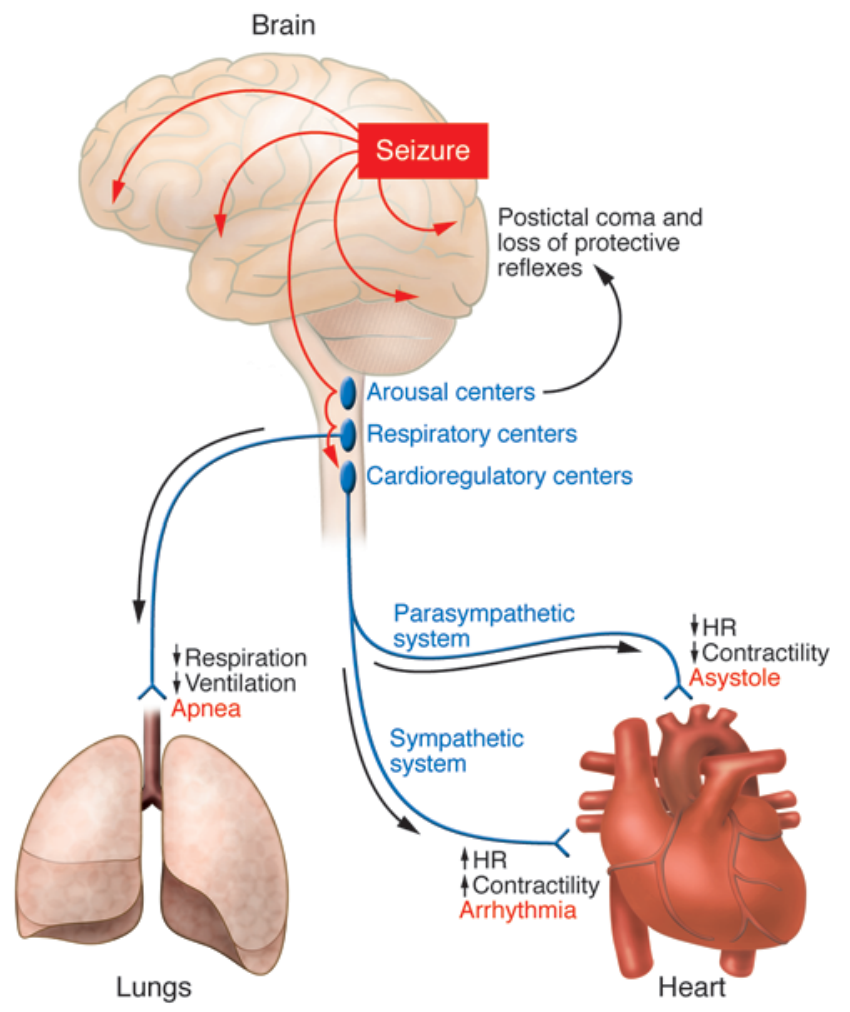

with epilepsy is whether or not the lessons of SUDEP in DS inform our understanding and prevention of SUDEP in the more than $99 \%$ of epilepsy patients without DS.

Address correspondence to: Orrin Devinsky, 223 East 34th St. New York, New York 10016, USA. Phone: 646.558.0803; Fax: 646.385.7164; E-mail: od4@nyu.edu.

1. Devinsky O. Sudden, unexpected death in epilepsy. NEngl J Med. 2011;365(19):1801-1811.
2. Sillanpaa M, Shinnar S. Long-term mortality in childhood-onset epilepsy. $N$ Engl J Med. 2010;363(26):2522-2529.

3. US Census. Table 120. Deaths and death rates by selected causes: 2006 and 2007. In: U.S. Census Bureau, Statistical Abstract of the United States: 2012. Washington, DC, USA: US Census Bureau; 2012:89.

4. Hesdorffer DC, et al. Combined analysis of risk factors for SUDEP. Epilepsia. 2011;52(6):1150-1159.

5. Ryvlin $P$, Rheims S. Increased risk of SUDEP among epileptic patients receiving placebo in randomised controlled trials. Epilepsia. 2009;50(s11):223.

6. Skluzacek JV, Watts KP, Parsy O, Wical B, Camfield P. Dravet syndrome and parent associations: the

\section{Figure 1}

Schematic of proposed mechanisms of seizure-associated death in SUDEP. Seizures originate in cortical structures and can propagate to brainstem nuclei involved in the regulation of arousal (e.g., periaqueductal gray area), cardioregulatory reflexes (e.g., nucleus of the solitary tract), and respiratory nuclei in the ventromedial medulla and caudal raphe. Seizure-related activation and postictal suppression of these regions can lead to the following: (a) postictal coma, which can lead to loss of protective airway reflexes; (b) increased sympathetic activation of the heart and associated increased risk of arrhythmia or parasympathetic activation and associated risk of asystole; (c) decreased respiration drive and hypoventilation. Severe seizurerelated derangements (indicated in red), either alone or in combination, would lead to death under the right environmental circumstances. HR, heart rate.
IDEA League experience with comorbid conditions, mortality, management, adaptation, and grief. Epilepsia. 2011;52(suppl 2):95-101.

7. Kalume F, et al. Sudden unexpected death in a mouse model of Dravet syndrome. J Clin Invest. 2013;123(4):1798-1808.

8. Schuele SU, Bermeo AC, Locatelli E, Burgess RC, Luders HO. Ictal asystole: a benign condition? Epilepsia. 2008;49(1):168-171.

9. Poh MZ, et al. Autonomic changes with seizures correlate with postictal EEG suppression. Neurology. 2012;78(23):1868-1876.

10. Richerson GB, Buchanan GF. The serotonin axis: Shared mechanisms in seizures, depression, and SUDEP. Epilepsia. 2011;52(suppl 1):28-38. 